\title{
Tamanho da amostra foliar para avaliação do estado nutricional e índice SPAD de capim-elefante irrigado para corte ${ }^{1}$
}

\author{
Leaf size sample to nutritional state and SPAD index evaluation in elephant grass \\ irrigated under cuts management
}

\section{SOUZA, Henrique Antunes de ${ }^{2 *}$; MODESTO, Viviane Cristina ${ }^{3}$; POMPEU, Roberto Cláudio Fernandes Franco ${ }^{2}$; NATALE, William ${ }^{4}$}

\author{
${ }^{1}$ Suporte financeiro Embrapa. \\ ${ }^{2}$ Embrapa Caprinos e Ovinos, Sobral, Ceará, Brasil.. \\ ${ }^{3}$ Universidade Estadual de São Paulo, Programa de Pós-Graduação em Zootecnia, Jaboticabal, São \\ Paulo, Brasil. \\ ${ }^{4}$ Universidade Estadual de São Paulo, Faculdade de Ciências Agrárias e Veterinária, Jaboticabal, São \\ Paulo, Brasil. \\ *Endereço para correspondente: henrique.souza@embrapa.br
}

\section{RESUMO}

A quantificação de unidades amostrais que irão compor a amostra promoverá a otimização da mão de obra, além de diminuir erros inerentes à recomendação e avaliação do estado nutricional. A coleta de folhas de forrageiras para a diagnose do estado nutricional é uma etapa importante para subsidiar a adubação, sendo o tamanho da amostra imprescindível para representar adequadamente a população de plantas a ser manejada. Assim, objetivou-se dimensionar o número de amostras de folhas para a diagnose do estado nutricional de capim-elefante irrigado para corte, bem como o índice de clorofila SPAD (Soil Plant Analysis Development). A avaliação foi realizada em capineira de capim-elefante, var. Cameroon, com irrigação fixa de baixa aspersão, em área localizada em Sobral-CE, em solo Neossolo fluvico. Coletaram-se 40 amostras simples, em zigue-zague, de maneira aleatória, escolhendo-se folhas recém-expandidas. As variáveis mensuradas foram os teores foliares de macro $(\mathrm{N}, \mathrm{P}, \mathrm{K}, \mathrm{Ca}, \mathrm{Mg}$ e S) e micronutrientes (B, $\mathrm{Cu}, \mathrm{Fe}, \mathrm{Mn}$ e $\mathrm{Zn}$ ) e o índice indireto de clorofila, com auxílio de um clorofilômetro. Considerando aceitável um erro amostral de $10 \%$, a coleta de amostras simples de 16 e 80 plantas, respectivamente, para macro e micronutrientes em capim-elefante seria adequada. Para a medida indireta da clorofila, 8 amostras são necessárias para obter um erro de $10 \%$.

Palavras-chave: Pennisetum purpureum, amostragem de folhas, nutrição de plantas

\section{SUMMARY}

The determination of the amount of sample units that will compose the sample express the optimization of the workforce, and reduce errors inherent in the report of recommendation and evaluation of nutritional status. The leaf collection for diagnosis of nutritional status is an important step, and the sample size necessary to adequately represent the population to be assessed. Thus, the objective was evaluation to the number simple design of leaf samples for the diagnosis of nutritional status of elephant grass irrigated for cutting and SPAD (Soil Plant Analysis Development) index. The study was conducted in elephant grass, with a low fixed sprinkler irrigation, in an area located in Sobral-CE in alluvial soil. It was collected 40 samples simple, randomly, choosing newly expanded leaves. The variables measured were foliar concentrations of macro (N, P, K, Ca, Mg and $\mathrm{S}$ ) and micronutrients $(\mathrm{B}, \mathrm{Cu}, \mathrm{Fe}, \mathrm{Mn}$ and $\mathrm{Zn}$ ) and the indirect index of chlorophyll, using a chlorophyll meter. Considering an acceptable error of $10 \%$, the collection of single samples of 16 and 80 plants, respectively, for macronutrients and micronutrients in elephant grass would be appropriate. For the indirect measurement of chlorophyll (SPAD index) 8 plants would be required to obtain a $10 \%$ error.

Keywords: Pennisetum purpureum, leaf sampling, plant nutrition 


\section{INTRODUÇÃO}

Entre as gramíneas tropicais, o capimelefante merece destaque tendo em vista a alta produtividade e a qualidade da forragem produzida. Dentre os fatores limitantes à cultura, evidencia-se o pouco conhecimento sobre as exigências nutricionais para $o$ estabelecimento e a manutenção da capacidade produtiva. No Brasil, são conhecidas muitas cultivares de capimelefante, tanto para corte como para pastejo, sobressaindo-se o Napier, o Cameroon, o Roxo, entre outras (MARTINS \& FONSECA, 1999). Ainda, estudos indicam a importância da adubação na melhoria da composição químico-bromatológica em capimelefante (OLIVEIRA et al., 2011).

De acordo com Boaretto et al. (2009), a análise química de plantas tem servido de instrumento para a divulgação de tecnologias que visam à produção econômica de culturas. Silva (2008) cita que a avaliação do teor de nutrientes nas folhas é utilizada como parâmetro para a avaliação do estado nutricional das plantas. Empregando-se essa técnica estabelece-se a correlação entre os dados de amostragem e os respectivos valores de referência para a cultura. $\mathrm{O}$ autor destaca, ainda, os fatores que interferem na avaliação do estado nutricional como a posição da folha, a parte da folha amostrada, a idade, o estágio fenológico da planta e o número de amostras.

Para a avaliação do estado nutricional do capim-elefante, Martinez et al. (1999) recomendam a coleta de 30 folhas expandidas, ou retiradas de todas as posições na parte aérea. Werner et al. (1997) não citam a quantidade de amostras à serem coletadas, apenas o período de amostragem que deve ser na fase de crescimento de brotações novas e folhas verdes. Quando se considera a avaliação do índice SPAD (Soil Plant Analysis Development), o qual apresenta estreita relação com o teor foliar de $\mathrm{N}$, não há informações disponíveis sobre o número de amostras a serem analisadas.

Hernandes et al. (2011), Rozane et al. (2007) e Rozane et al. (2009) relatam o número ideal de amostras simples de plantas para compor uma amostra composta para a avaliação do estado nutricional, mas a maioria desses trabalhos avalia plantas perenes, como as frutíferas.

Diante do exposto, este trabalho foi conduzido com o objetivo de dimensionar o número de amostras adequadas para realizar a diagnose do estado nutricional e índice SPAD do capim-elefante irrigado para corte.

\section{MATERIAL E MÉTODOS}

O estudo foi realizado no município de Sobral-CE, em 4 hectares de capineira de capim-elefante var. Cameroon durante o primeiro semestre de 2012, em área pertencente a Embrapa Caprinos e Ovinos. A forrageira apresenta cerca de 30 anos de idade da implantação da área, tendo sido renovada aproximadamente a 10 anos, irrigada por aspersão fixa de baixa pressão, altitude de $100 \mathrm{~m}$ e o solo da área é tipo Neossolo Fluvico. O corte do capim é realizado a cada 60 dias (ciclo). A análise de solo da área está apresentada na Tabela 1.

Segundo Raij et al. (1997) os valores para fósforo, potássio, cálcio, magnésio, boro, cobre, ferro, manganês e zinco estão dentro das classes: médio, muito alto, alto, alto, médio, médio, alto, alto e médio respectivamente. De acordo com Werner et al. (1997), a saturação por bases para o capim-elefante está adequada. 
Rev. Bras. Saúde Prod. Anim., Salvador, v.15, n.3, p.584-591 jul./set., 2014 http://www.rbspa.ufba.br ISSN 15199940

Tabela 1. Análise química de solo da área experimental

\begin{tabular}{cccccccccc}
\hline $\mathrm{pH}\left(\mathrm{CaCl}_{2}\right)$ & $\mathrm{M} . \mathrm{O}$. & $\mathrm{P}_{\text {resina }}$ & $\mathrm{K}$ & $\mathrm{Ca}$ & $\mathrm{Mg}$ & $\mathrm{H}+\mathrm{Al}$ & $\mathrm{SB}$ & $\mathrm{T}$ & $\mathrm{V}$ \\
\hline & $\mathrm{g} \mathrm{dm}^{-3}$ & $\mathrm{mg} \mathrm{dm}^{-3}$ & & \multicolumn{7}{c}{$\mathrm{mmol}_{\mathrm{c}} \mathrm{dm}^{-3}$} \\
\hline 5,4 & 20 & 24 & 5,5 & 44 & 24 & 31,0 & 73,5 & 104,5 & 70 \\
\hline $\mathrm{B}$ & $\mathrm{Cu}$ & $\mathrm{Fe}$ & $\mathrm{Mn}$ & $\mathrm{Zn}$ & - & - & - & - & - \\
\hline \multicolumn{2}{c}{$\mathrm{mg} \mathrm{dm}^{-3}$} & & & & - & - & - & - & - \\
\hline 0,45 & 0,5 & 49 & 27,5 & 0,6 & - & - & - & - & - \\
\hline
\end{tabular}

No segundo semestre de 2011, foi realizada a aplicação em um único ciclo das seguintes quantidades de $\mathrm{N}$ (ureia), $\mathrm{P}_{2} \mathrm{O}_{5}$ (superfosfato triplo) e $\mathrm{K}_{2} \mathrm{O}$ (cloreto de potássio): 90; 43; e $120 \mathrm{~kg}$ $\mathrm{ha}^{-1}$ respectivamente. Aplicou-se, ainda, $25 \mathrm{~kg} \mathrm{ha}^{-1}$ de FTE (BR-12) e $2 \mathrm{t} \mathrm{ha}^{-1} \mathrm{de}$ esterco de ovino curtido. Nos ciclos seguintes foram efetuadas aplicações de $50 \mathrm{~kg} \mathrm{ha}^{-1}$ de N, utilizando a ureia como fonte deste nutriente. As aplicações foram realizadas em área total, com o auxílio do implemento distribuidor de agregados (esparramador).

Para a diagnose do estado nutricional e a avaliação do índice SPAD do capimelefante, coletaram-se 40 amostras simples (perfilhos), em zigue-zague, de maneira aleatória, em 1 hectare, amostrando-se folhas recém-expandidas (WErner et al., 1997). A amostragem foi realizada em um ciclo quando as plantas estavam aptas para o corte, com aproximadamente 55-60 dias e altura variando entre 1,50 e $1,65 \mathrm{~m}$. Após a coleta das folhas, o índice SPAD foi mensurado no meio do limbo foliar e evitando-se a nervura central, a determinação foi procedida antes da lavagem e utilizando-se o clorofilômetro Minolta SPAD-502. O material vegetal foi lavado e seco em estufa de ventilação forçada de ar a $65{ }^{\circ} \mathrm{C}$, até atingir massa constante. As amostras secas foram moídas em moinho tipo Wiley, para posterior análise química de macro e micronutrientes, conforme descrito em Bataglia et al. (1983).
Com base nos dados de teores de nutrientes e do índice SPAD, o número de unidades amostrais para estimar os parâmetros de uma população infinita para um nível de precisão desejado, baseando-se no erro-padrão da média, foi dado pela equação descrita conforme Thompson (1992):

$$
n=\frac{t^{2} s^{2}}{d^{2} m^{2}}
$$

Na qual: $n$ é o tamanho da amostra estimada; $t$ é o valor da distribuição $t$ de Student a $5 \%$ de probabilidade; $s^{2}$ é a variância; $d$ é o erro na estimativa da média, em porcentagem, variando de 1 a $30 \%$ de $m ; m$ é a média amostral.

\section{RESULTADOS E DISCUSSÃO}

A Tabela 2 apresenta a análise estatística descritiva, referente aos dados de macronutrientes e do índice SPAD e a estimativa do número de unidades amostrais necessárias para compor a amostra representativa da capineira. No caso, a quantidade de unidades amostrais necessárias para formar amostra composta está condicionada ao nível de precisão desejado. Com a elevação do erro amostral seria necessário menor número de plantas amostradas, sendo que com o aumento de plantas mensuradas houve diminuição do erro, ou seja, a estimativa do número de unidades amostrais 
necessárias para compor a amostra diminui à medida que se aumenta o $d$ (Tabela 2).

Comparou-se os valores médios presentes na Tabela 2 com as faixas de nutrientes consideradas adequadas por Werner et al. (1997) para capimelefante (pastejo), verificando-se que $\mathrm{P}$, $\mathrm{K}, \mathrm{Ca}, \mathrm{Mg}$ e $\mathrm{S}$ encontram-se na faixa adequada e, apenas o $\mathrm{N}$ está abaixo.
Admitindo-se um erro aceitável de 10\%, 16 plantas de capim-elefante seriam suficientes para a análise de macronutrientes (Tabela 2). A variabilidade dos atributos analisados, estimado pelo número de amostras simples de plantas necessárias para compor uma amostra composta, seguiu a seguinte ordem: $\mathrm{K}<\mathrm{N}<$ Índice $\mathrm{SPAD}<\mathrm{Mg}<\mathrm{Ca}<\mathrm{P}<\mathrm{S}$.

Tabela 2.Estatística descritiva e estimativa do número de plantas necessárias (unidades amostrais) a compor amostra para a avaliação dos teores de macronutrientes e do índice SPAD, em função do erro na estimativa da média

\begin{tabular}{lccccccc}
\hline Erro na estimativa da & $\mathrm{N}$ & $\mathrm{P}$ & $\mathrm{K}$ & $\mathrm{Ca}$ & $\mathrm{Mg}$ & $\mathrm{S}$ & Índice \\
\hline 1 & 668 & 1592 & 418 & 1423 & 960 & 1612 & 784 \\
5 & 27 & 64 & 17 & 57 & 38 & 64 & 31 \\
10 & 7 & 16 & 4 & 14 & 10 & 16 & 8 \\
15 & 3 & 7 & 2 & 6 & 4 & 7 & 3 \\
20 & 2 & 4 & 1 & 4 & 2 & 4 & 2 \\
30 & 1 & 2 & 0 & 2 & 1 & 2 & 1 \\
\hline \multicolumn{7}{c}{$\mathrm{g} \mathrm{kg}^{-1}$} \\
\hline$m$ & 13,9 & 2,0 & 18,6 & 4,1 & 2,0 & 1,0 & 28,1 \\
$s$ & 1,8 & 0,4 & 1,9 & 0,8 & 0,3 & 0,2 & 3,9 \\
$s(m)$ & 0,3 & 0,1 & 0,3 & 0,1 & 0,0 & 0,0 & 0,6 \\
\hline CV $(\%)$ & 12,8 & 19,7 & 10,1 & 18,6 & 15,3 & 19,8 & 13,8 \\
\hline$m$ é a estimativa da média amostral; $s$ é o desvio-padrão; $s(m)$ é o erro-padrão da média; e CV é o \\
coeficiente de variação. $\mathrm{n}=40$.
\end{tabular}

Em estudo de dimensionamento de número de amostras foliares Rozane et al. (2009) e Hernandes et al. (2011) consideraram um erro aceitável entre 5 e $10 \%$. Quando o objeto de estudo é quantidade de amostra simples para avaliação da fertilidade do solo, Guarçoni et al. (2006) e Rozane et al. (2011) consideraram erro aceitável entre 10 a $20 \%$. Logo, pode-se considerar que para avaliação da diagnose foliar a tolerância aceitável é menor.

Avalhães et al. (2009) estudaram os sintomas da omissão de macronutrientes em plantas de capim-elefante cv. Mott em solução nutritiva, observando que a omissão de $\mathrm{N}, \mathrm{P}, \mathrm{K}, \mathrm{Ca}, \mathrm{Mg}$ e $\mathrm{S}$ limitaram a produção de massa seca da parte aérea dessa gramínea, em relação ao tratamento completo. A deficiência de um macronutriente, além de provocar redução do seu teor na parte aérea, diminuiu o acúmulo da maioria dos macronutrientes, com consequentes alterações morfológicas, traduzidas como sintomas característicos de deficiência.

Ressalta-se, ainda, que para a determinação da medida indireta da clorofila (índice SPAD), que apresenta estreita relação com $\mathrm{o}$ teor de nitrogênio, a amostragem de 8 plantas 
em diferentes touceiras seria suficiente para obter um erro aceitável de 5 a $10 \%$, valor este, próximo ao observado para o nitrogênio (de sete amostras). Araújo et al. (2013) verificaram que o índice SPAD pode ser utilizado para avaliação rápida e não destrutiva da clorofila em capim-elefante.

Segundo Rozane et al. (2007), para a avaliação do estado nutricional de macronutrientes em mangueiras, apenas 10 plantas seriam suficientes para obterse um erro aceitável de 5 a $10 \%$. Hernandes et al. (2011) verificaram que 21 plantas seriam necessárias para as determinações químicas foliares de macronutrientes em caramboleiras, considerando um erro amostral de $10 \%$.
O acompanhamento do estado nutricional é determinante para o manejo adequado da fertilização. Dessa forma, Pegoraro et al. (2009) observaram que o manejo da irrigação e da adubação nitrogenada em capimelefante propiciaram maior produção de biomassa da parte aérea, o que determinou maiores acúmulos e exportações de $\mathrm{K}$, Ca e P pela planta.

A Tabela 3 apresenta a análise estatística descritiva dos dados de micronutrientes. De maneira análoga aos resultados de macronutrientes, com o maior número de plantas coletadas o erro foi menor, ou seja, há diminuição do erro com o aumento do número de folhas dos perfilhos amostrados.

Tabela 3. Estatística descritiva e estimativa do número de plantas necessárias (unidades amostrais) a compor amostra para a avaliação dos teores de micronutrientes, em função do erro na estimativa da média

\begin{tabular}{|c|c|c|c|c|c|}
\hline Erro na estimativa da média $(d), \%$ & $\mathrm{~B}$ & $\mathrm{Cu}$ & $\mathrm{Fe}$ & $\mathrm{Mn}$ & $\mathrm{Zn}$ \\
\hline 1 & 2514 & 1093 & 879 & 7974 & 1250 \\
\hline 5 & 101 & 44 & 35 & 319 & 50 \\
\hline 10 & 25 & 11 & 9 & 80 & 12 \\
\hline 15 & 11 & 5 & 4 & 35 & 6 \\
\hline 20 & 6 & 3 & 2 & 20 & 3 \\
\hline 30 & 3 & 1 & 1 & 9 & 1 \\
\hline & \multicolumn{5}{|c|}{$\mathrm{mg} \mathrm{kg}^{-1}$} \\
\hline$m$ & 13,9 & 4,7 & 40,4 & 38,6 & 24,3 \\
\hline$s$ & 3,5 & 0,8 & 5,9 & 17,0 & 4,2 \\
\hline$s(m)$ & 2,0 & 2,0 & 2,0 & 2,0 & 2,0 \\
\hline $\mathrm{CV}(\%)$ & 24,8 & 16,3 & 14,7 & 44,1 & 17,5 \\
\hline
\end{tabular}

$m$ é a estimativa da média amostral; $s$ é o desvio-padrão; $s(m)$ é o erro-padrão da média; e CV é o coeficiente de variação. $n=40$.

Em relação às faixas de teores de micronutrientes, propostas por Werner et al. (1997) como adequadas para capim-elefante sob pastejo, observou-se que o B está abaixo do adequado, e $\mathrm{Cu}$, $\mathrm{Fe}, \mathrm{Mn}$ e $\mathrm{Zn}$ estão na faixa considera adequada, respectivamente.

Admitindo-se um erro aceitável de 5 a $10 \%, 80$ plantas de capim-elefante seriam necessárias para as análises de micronutrientes (Tabela 3). A variabilidade dos atributos avaliados, estimado pelo número de amostras simples de plantas necessárias para compor uma amostra composta, seguiu a seguinte ordem: $\mathrm{Fe}<\mathrm{Cu}<\mathrm{Zn}<\mathrm{B}<\mathrm{Mn}$.

Outros trabalhos também verificaram alta variabilidade para a determinação de micronutrientes, como verificado por Rozane et al. (2007), Rozane et al. 
(2009) e Hernandes et al. (2011). As justificativas destes autores estão relacionadas às aplicações foliares de micronutrientes e ao tipo de solo; no entanto, no presente estudo, houve aplicação de micronutrientes, a qual foi realizada acompanhada da adubação de manutenção em área total.

Vários estudos foram realizados com fins de verificar o número de amostras adequadas, seja para atributos químicos ou físicos do solo (GUARÇONI et al., 2007; TAVARES FILHO \& RIBON, 2008; OLIVEIRA et al., 2007; SANTOS et al., 2008), caracteres fitotécnicos (CARGNELUTTI FILHO et al., 2008; CARGNELUTTI et al., 2009; Cargnelutti et al., 2010; HAESBAERT et al., 2011; SANTOS et al., 2010; SOUZA et al., 2002), no entanto, trabalhos que relatem o dimensionamento amostral para diagnose foliar, e principalmente de forrageiras são escassos.

Para as determinações químicas dos macronutrientes e micronutrientes, a coleta de amostras simples de 4 e 20 plantas, respectivamente, de capimelefante seriam suficientes, considerando um erro aceitável de $20 \%$. Para a medida indireta da clorofila (índice SPAD) a coleta de folhas em 8 perfilhos seria necessária para um erro de $10 \%$.

\section{REFERÊNCIAS}

ARAÚJO, S.A.C.; VASQUEZ, H.M.; TORRES NETTO, A.; CAMPOSTRINI, E.; DEMINICIS, B.B.; LIMA, E.S. Indirect method for quantifying the content of photosynthetic pigments in genotypes of dwarf elephant grass. Acta

Scientiarum. Animal Sciences, v.35, p.43-47, 2013.
AVALHÃES, C.C.; PRADO, R.M.; ROZANE, D.E.; ROMUALDO, L.M.; CORREIA, M.A.R. Omissão de macronutrientes no crescimento e no estado nutricional de capim-elefante (cv. Mott) cultivado em solução nutritiva. Scientia Agraria, v.10, p.215-222, 2009.

BATAGLIA, O.C.; FURLANI, A.M.C.; TEIXEIRA, J.P.F.; FURLANI, P.R.; GALLO, J.R. Métodos de análise química de plantas. Campinas: Instituto Agronômico de Campinas, 1983. 48p. (Boletim Técnico, 78).

BOARETTO, A.E.; RAIJ, B. van; SILVA, F.C.; CHITOLINA, J.C.; TEDESCO, M.J.; CARMO, C.A.F.S. Amostragem, acondicionamento e preparo de amostras de plantas para análise química. In: SILVA, F. Manual de análises químicas de solos, plantas e fertilizantes. Brasília: Embrapa Informação Tecnológica, 2009. p.59-86.

CARGNELUTTI FILHO, A.; RIBEIRO, N. D.; STORCK, L.; JOST, E.; POERSCH, N. L. Tamanho de amostra de caracteres de cultivares de feijão. Ciência Rural, v.38, p.635-642, 2008.

CARGNELUTTI FILHO, A.; EVANGELISTA, D.H.R.; GONÇALVES, E.C.P.; STORCK, L. Tamanho de amostras de caracteres de genótipos de soja. Ciência Rural, v.39, p.983-991, 2009.

CARGNELUTTI FILHO, A.; LOPES, S.J.; BRUM, B.; SILVEIRA, T.R.; TOEBE, M.; STORCK, L. Tamanho de amostras de caracteres em híbridos de mamoneira. Ciência Rural, v.40, p.280-287, 2010. 
Rev. Bras. Saúde Prod. Anim., Salvador, v.15, n.3, p.584-591 jul./set., 2014 http://www.rbspa.ufba.br ISSN 15199940

GUARÇONI, M.A.; ALVAREZ V., V.H.; NOVAIS, R.F.; CANTARUTTI, R.B.; LEITE, H.G.; FREIRE, F.M.

Definição da dimensão do indivíduo solo e determinação do número de amostras simples necessário à sua representação. Revista Brasileira de Ciência do Solo, v.30, p.943-954, 2006.

GUARÇONI, M.A.; ALVAREZ V.V.H.; NOVAIS, R.F.; CANTARUTTI, R.B.; LEITE, H.G.; FREIRE, F.M. Diâmetro de trado necessário à coleta de amostras num Cambissolo sob plantio direto ou sob plantio convencional antes ou depois da aração. Revista Brasileira de Ciência do Solo, v.31, p.947-959, 2007.

HAESBAERT, F.M.; SANTOS, D.; LÚCIO, A.D.; ANTONELLO, V.B.B.I.; RIBEIRO, A. L. P. Tamanho de amostra para experimentos com feijão-de-vagem em diferentes ambientes. Ciência Rural, v.41, p.38-44, 2011.

HERNANDES, A.; ROZANE, D.E.; SOUZA, H.A.; ROMUALDO, L.M.; NATALE, W. Amostragem para diagnose do estado nutricional e avaliação da fertilidade do solo em caramboleiras. Bragantia, v.70, p.657-663, 2011.

MARTINS, C.E.; FONSECA, D.M. Manejo de solo e adubação de pastagem de capim-elefante. In: PASSOS, L.P.; CARVALHO, L.A. C.; MARTINS, C.E.; BRESSAN, M.; PEREIRA, A.V. Biologia e manejo do capim-elefante. Juiz de Fora: Embrapa-CNPGL, 1999. p.83-118.

MARTINEZ, H.E.P.; CARVALHO, J.G.; SOUZA, R.B. Diagnose foliar. In: RIBEIRO, A.C.; GUIMARÃES, P.T.G.; ALVAREZ, V.V.H. Recomendações para o uso de corretivos e fertilizantes em Minas Gerais. $5^{\text {a }}$ Aproximação. Viçosa: Universidade Federal de Viçosa, 1999. p.143-168.
OLIVEIRA, F.H.T.; ARRUDA, J.A.; SILVA, I.F.; ALVES, J.C. Amostragem para a avaliação da fertilidade do solo em função do instrumento de coleta das amostras e de tipos de preparo do solo. Revista Brasileira de Ciência do Solo, v.31, p.973-983, 2007.

OLIVEIRA, T.S.; PEREIRA, J.C.; REIS, C.S.; QUEIROZ, A.C.; CECON, P.R.; GOMES, S.T. Composição químicobromatológica do capim-elefante submetido à adubação química e orgânica. Revista Brasileira de Saúde e Produção Animal [online], v.12, p.3242, 2011.

PEGORARO, R.F.; MISTURA, C.; WENDLING, B.; FONSECA, D.M.; FAGUNDES, J.L. Manejo da água e do nitrogênio em cultivo de capim-elefante. Ciência e Agrotecnologia, v.33, p.461467, 2009.

RAIJ, B. van; CANTARELLA, H.; QUAGGIO, J.A.; FURLANI, A.N.C.

Recomendações de adubação e calagem para o Estado de São Paulo.

Campinas: Instituto

Agronômico/Fundação IAC, 1997. 285p.

(Boletim Técnico, 100).

ROZANE, D.E.; NATALE, W.; PRADO, R.M.; BARBOSA, J.C. Amostragem para diagnose do estado nutricional de mangueiras. Revista Brasileira de Fruticultura, v.29, p.371376, 2007.

ROZANE, D.E.; NATALE, W.; PRADO, R.M.; BARBOSA, J.C. Tamanho da amostra foliar para avaliação do estado nutricional de goiabeiras com e sem irrigação. Revista Brasileira de Engenharia Agrícola e Ambiental, v.13, p.233-239, 2009. 
ROZANE, D.E.; ROMULADO, L.M.; CENTURION, J.F.; BARBOSA, J.C. Dimensionamento do número de amostras para avaliação da fertilidade do solo. Semina: Ciências Agrárias, v.32, p.111-118, 2011.

SANTOS, H.C.; OLIVEIRA, F.H.T.; ARRUDA, J.A.; LOPES, A.R.S.; SOUZA JÚNIOR, R.F.; FARIAS, D.R. Amostragem para avaliação da fertilidade do solo em função da variabilidade de suas características químicas. Revista Brasileira de Engenharia Agrícola e Ambiental, v.13, p.849-854, 2009.

SANTOS, D.; HAESBAERT, F.M.; PUHL, O.J.; SANTOS, J.R.A.; LÚCIO, A.D. Suficiência amostral para alface cultivada em diferentes ambientes.

Ciência Rural, v.40, p.800-805, 2010.

SILVA, M.A.C. Importância dos critérios de amostragem de folhas. In: PRADO, R.M.; ROZANE, D.E.; VALE, D. W.; CORREIA, M.A. V.; SOUZA, H.A. Nutrição de plantas: diagnose foliar em grandes culturas. Jaboticabal: FCAV/Capes/Fundunesp, 2008. p.61-82.
SOUZA, M.F.; LÚCIO, A.D.; STORCK, L.; CARPES, R.H.; SANTOS, P.; SIQUEIRA, L.F. Tamanho da amostra para peso de massa de frutos, na cultura da abóbora italiana em estufas plásticas. Revista Brasileira de Agrociência, v.8, p.123128, 2002.

TAVARES FILHO, J.; RIBON. A.A. Resistência do solo à penetração em reposta ao número de amostras e tipo de amostragem. Revista Brasileira de Ciência do Solo, v.32, p.487-494, 2008.

THOMPSON, S.K. Sampling. New York: John Wiley, 1992. 343p.

WERNER, J.C.; PAULINO, V.T.; CANTARELLA, H.; QUAGGIO, J.A.; ANDRADE, N.O. Forrageiras. In: RAIJ, B. van; CANTARELLA, H.; QUAGGIO, J.A.; FURLANI, A.N.C.

Recomendações de adubação e calagem para o Estado de São Paulo. Campinas: Instituto Agronômico, 1997. 285 p. (Boletim Técnico, 100).

Data de recebimento: 20/10/2013

Data de aprovação: 19/09/2014 